The Breathe feature where we give you an expert and a topic, and you have the chance to ask them any questions you wish viabreathe@ersj.org.uk

\title{
Ask the Expert: Clinical dilemmas in asthma
}

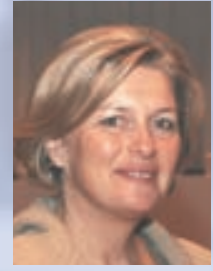

M. Gaga

7th Respiratory Medicine Dept and Asthma Centre "Sotiria" Hospital Athens Chest Hospital 152 Mesogion Avenue Athens 11527

Greece

Fax: 302107781911

E-mail:mgaga@med.uoa.gr

\section{Competing interests} None declared
1. What is your opinion of the role of subcutaneous terbutaline delivered via a syringe pump to patients with severe asthma (with normal immunoglobulin E) who are unable to come down below a moderate dose of oral prednisolone $(20 \mathrm{mg})$ without becoming symptomatic or having multiple exacerbations?

I. Chakravorty, Stevenage, UK

A

1. There is only one published study in the literature showing that some but not all children with severe asthma showed benefit [1]. This was an open study, therefore a placebo effect cannot be ruled out, and it is only one study examining $~ 10$ children. Prof. A. Bush from the Royal Brompton Hospital (London, UK) is currently conducting a double-blind study, so within the next year we will have evidence from one properly conducted study. I think the patient's asthma phenotype may help us understand which individuals might benefit from constant bronchodilatation. If a patient has sputum eosinophilia and frequent exacerbations, I would certainly insist on high-dose steroids; I believe that those patients are at risk from a reduction of their steroid dose combined with an increased dose of bronchodilatation. If on the other hand, the patient has no eosinophilia and no frequent exacerbations but is handicapped in his everyday activity by breathlessness, I might consider a short trial under very close monitoring and I would probably do it with the patient hospitalised. I think inhaled $\beta_{2}$-agonists, whether inhaled or nebulised, work very well in the nonacute severe setting and that systemic dosing has more side-effects.

2. What is your view of endobronchial thermoplasty in asthma and its future role in the management of asthma?

V. Deshmukh, Mumbai, India

2. The first studies using bronchial thermoplasty $[2,3]$ seemed quite promising; however more recently, newer data have shown that the intervention is less promising in the long term and there may be adverse effects during the procedure. I think that it should only be used in specifically selected patients, and probably as part of a research programme.

3. I practice in a tertiary centre in India. Most of the patients I see have moderate-to-severe persistent asthma. Despite being on all available drugs (long-acting $\beta$-agonist plus inhaled corticosteroids maximum permissible dose, sustained-release theophylline and montelukast), many of these patients never seem to achieve normal/best peak expiratory flow (PEF) or forced expiratory volume in $1 \mathrm{~s}$ (FEV 1 ) - although they do not have symptoms of acute exacerbation. When given a short course of systemic steroids, these patients seem to show a significant improvement in signs and symptoms with normalisation of PEF and/or FEV1. However, the effect of this short burst of steroids seems to wear off over the next 4-6 weeks and the patients again show a gradual decline in PEF to previous levels, although they may or may not be symptomatic. As quite a few of these patients are middle-aged individuals (aged 30-50 years) who lead an active life, they complain of limitation in exercise capacity. The first dilemma is whether to put these patients on regular low-dose maintenance systemic steroids for a long period of time, in an effort to preserve lung function for the future (but obviously exposing them to the side-effects of long term steroids) or to compromise and 
accept low PEF and exercise limitation while protecting them from the side-effects of systemic steroids, risking decline in lung function over the next few decades.

The other dilemma is that, because of poor perception or because of limited physical activity, some patients do not complain of significant symptoms although they have a very low PEF or FEV1, again despite being on maximum therapy. How justifiable is it to treat patients who are not symptomatic with long-term systemic steroids in an effort to preserve lung function?

Omalizumab is an extremely expensive option and steroid-sparing therapies such as methotrexate do not seem to work very well!

Should we in such cases think of long-term issues such as airway remodelling and compromised lung function in the future and give systemic steroids or should we explain to the patient that they will have to accept exercise limitation and a few symptoms here and there to protect themselves from the side-effects of toxic therapies in the long run?

H. Dumra, Ahmedabad, India

3. These are dilemmas we all face. To begin with, if patients indeed improve on high-dose oral steroids and the effect wears off fairly quickly so that they have to have a new course every 4-6 weeks, I would certainly treat them with a longer course of oral steroids and with a higher inhaled steroid dose. I agree there is no evidence, but there are published studies from specialised asthma centres reporting median inhaled corticosteroid doses of 3,500 $\mu \mathrm{g}$ [4]. If there are no troublesome local effects, the side-effects are certainly lower than with systemic steroids. Prior to prescribing high doses, I would check again their compliance, their exposure to factors at work or home (mould, dust, smoke) and comorbidities.

With patients who do not suffer exacerbations and do not complain of symptoms, I would not institute oral steroids to preserve their lung function: patients may face more morbidity and mortality from steroid side-effects than from asthma. In fact, there is no evidence showing that inhaled corticosteroids in asymptomatic patients prevent lung function decline and indeed, there is no good correlation between severity, duration of asthma and rate of decline in lung function.

The cost of omalizumab is indeed very high. In well-selected patients its use may be cost-effective but if your health system does not support its use, the cost cannot be met by most patients. And I agree that most studies on methotrexate are not encouraging. If you feel, however, that some of your patients need continuous high-dose oral corticosteroids, it is perhaps worth examining the effect of adding methotrexate.

Lastly, I strongly believe that any decision on treatment should indeed involve the patient. We should discuss with them our dilemmas, give them our view and come to a mutually acceptable decision. The dosing should not be lower than we think safe, nor higher than we find acceptable but within this dose range the patient can decide whether he/she wants less treatment or fewer symptoms.

4. I am a practicing chest physician in Nigeria. Here, we see asthmatic patients who mostly do not have Western-style education. There are problems relating to their asthma monitoring and drug treatment. Only a small number of our patients can afford inhaler medications regularly and of these only a few can use the inhalers correctly. They cannot carry out home monitoring of PEF. We are left with the option of oral medications and injectable drugs with their attendant adverse effects. What are your views on these dilemmas in asthma management?

\section{A. Hammangabdo, Maiduguri, Nigeria}

4. You are touching on important and difficult problems. There is a worldwide effort for the production of generics, but it is a sad fact that medications are still not affordable or available through some type of insurance/health plan all around the world. I think the only solution is lobbying with local governments and liaising with the World Health Organization (WHO) or other organisations for help because certainly inhaled corticosteroids have changed the face of asthma ever since beclomethasone became widely used. Generic beclomethasone comes quite 
cheap and it is unthinkable not to be able to use it. Indeed, the WHO has developed two initiatives: Practical Approach to Lung Health (PAL) and the Global Alliance Against Chronic Respiratory Diseases (GARD). The International Union Against Tuberculosis and Lung Diseases (The Union) has launched a new initiative to increase the affordability of essential asthma drugs for patients in developing countries termed the Asthma Drug Facility (ADF), which could facilitate the care of patients living in your part of the world. The ADF supplies the following inhaled drugs in CFC-free and CFC-containing formulations: salbutamol $100 \mu \mathrm{g}$, terbutaline $250 \mu \mathrm{g}$, beclomethasone $250 \mu \mathrm{g}$, budesonide $200 \mu \mathrm{g}$ and fluticasone $125 \mu \mathrm{g}$. Countries or organisations within countries can purchase affordable, good-quality essential medicines for asthma through the ADF and I hope that through Breathe, you will be given a contact address that will enable you to obtain affordable medications for your patients.

I believe that education regarding asthma is possible anywhere and I find that many of my lesseducated patients are better at understanding or complying. It is hard work requiring time, and where you work there are few doctors, but perhaps a nurse or other devoted individual could help. What is very important is to explain things to patients in a way they can understand. And even though PEF meters may not be available, a translation of the Asthma Control Test (ACT) or Asthma Control Questionnaire (ACQ) can help: it does with my patients. The use of inhalers can be improved with repeated demonstration, and possibly a paper cup or tubing to act as a spacer. It is not perfect but it is better than nothing.

5. Which parameters do you use when considering whether a patient is well controlled, and how should we evaluate these parameters (after 6 or 3 months)? In Brazil, exhaled nitric oxide (NO) measurement is too expensive and sputum is not well processed in many centres. Could small questionnaires be useful?

G.N. Yonezawa, São Paulo, Brazil

$\Delta$

5. NO and sputum induction are fairly expensive or time-consuming and are available only in a hospital setting, even in countries with advanced health systems. Questionnaires such as the ACQ or the ACT are very useful because they are standardised and quick to perform. Timing a reevaluation visit depends on the patient. A new patient with a severe exacerbation and a history of severe asthma should perhaps be evaluated after 2 weeks or 1 month at the latest. A patient with mild asthma with few symptoms and a mild history can be reviewed after 3 or 6 months. I tend to review new patients more often until I feel I know them. Then set appointments at 3 or 6 months but always ask them to contact you if they suffer an exacerbation.

6. a) Could you point out a practical and realistic diagnostic approach for patients with difficult-to-treat asthma, once the patient has a proven diagnosis of asthma and does not respond to conventional therapy? b) What kind of diagnostic studies are useful in deciding therapy for patients with difficult-to-treat asthma? c) Are asthma phenotypes useful in difficult-to-treat asthma from a practical and daily clinical practice point of view? Can you change your therapy based on these phenotypes?

\section{A. Gómez, San Luis Potosi, Mexico}

$\Delta$

6. A diagnostic re-evaluation of severe asthma includes tests to examine comorbidities, triggers, compliance and assessment of inflammation. Frequent comorbidities include psychopathology, rhinosinusitis, Chlamydia pneumonia infection, gastro-oesophageal reflux disease, diabetes and thyroid dysfunction. Frequent triggers include aspirin intake, noncompliance or forgetting to take medication, occupational exposure, mould in the house or exposure to pets. What you choose to do depends on your resources and the patient's history and symptoms. I think that at least a rough assessment of the patient's psychological profile, home environment and testing for comorbidities indicated by the history are important. Assessment of inflammation is important, at least initially, to help guide treatment. And yes, asthma phenotypes are important and help us in our decisions. A patient with sputum eosinophilia will probably benefit from increased steroid doses while a patient with breathlessness but no eosinophils mostly needs bronchodilators [5-7]. 
Q

7. I think that the Global Initiative for Asthma (GINA) recommendation on the spirometric diagnosis of asthma based on reversibility (change in $\mathrm{FEV} 1>200 \mathrm{~mL}$ and $>12 \%$ ) is totally wrong and leads to underdiagnosis. This is because when an asthmatic comes to a physician with a typical history but during a symptom-free day, he or she may have a normal baseline FEV1 (i.e. $3.5 \mathrm{~L}$ ) and, for instance, the improvement is $300 \mathrm{~mL}$ which is $<12 \%$. This example applies to a substantial number of patients, especially in countries such as Greece, where the majority of asthmatics lie on the mild spectrum of the disease. I believe that the previous version of GINA (change in FEV $1>200 \mathrm{~mL}$ or $>12 \%$ ) was correct and should be reconsidered. What is your opinion?

\section{E. Kosmas, Athens, Greece}

A

7. I think you are right in saying that it is more difficult to confirm a diagnosis of asthma in patients with high FEV 1 values. In a patient who gives a history compatible with asthma and who improves by $400 \mathrm{~mL}$, even if this is only $9 \%$ over his pre-bronchodilatation value, there is a high suspicion of asthma and I think most clinicians would consider it even if they cannot confirm it outright. Certainly, the absence of confirmation does not exclude this diagnosis and most physicians may, in such cases, consider a bronchial challenge, closer follow-up or a trial of steroids, depending on the setting. Conversely, if we used the version "change in $\mathrm{FEV} 1>200 \mathrm{~mL}$ or $>12 \%$ ", many chronic obstructive pulmonary disease patients (e.g. those having $<0.8 \mathrm{~L} \mathrm{FEV} 1$ and a reversibility of $100 \mathrm{~mL}$ ) would be considered asthmatic although they are not. I think that any definition used to confirm a diagnosis should include most of the positive cases but also exclude most of the negative ones and I feel that the $>200 \mathrm{~mL}$ or $>12 \%$ version may over diagnose asthma. Perhaps a version stating "change in $\mathrm{FEV} 1>200 \mathrm{~mL}$ and $>12 \%$ or change in FEV 1 $>300 \mathrm{~mL}$ and $>9 \% "$ or something along those lines might be worth considering in the future.

Q

8. Variable airway obstruction seems to be a hallmark of asthma. Which parameter can best be used in order to carefully detect variable airway obstruction in subjects who are able to perform the forced expiratory manoeuvre: PEF, FEV1 or FEV1/forced vital capacity (FVC) ratio? Is the choice age-dependent?

B. Ponsioen, Rotterdam, The Netherlands

$\Lambda$

8. I think most studies find FEV 1 more sensitive and more reproducible and it is the measurement of choice, while the ratio is also available through the same manoeuvre and helps us judge how severe the obstruction is. PEF is a good home measurement but actually the PEF rate manoeuvre is different from the FVC manoeuvre and it should be measured using a peak flow meter, especially if the spirometer does not measure the envelope curve. There is much more variability in PEF, which is best used to monitor a specific patient longitudinally rather than to evaluate lung function. Furthermore, many studies have shown that there is considerable variability between $\mathrm{FEV} I$ and $\mathrm{PEF}$, when expressed as \% values. I am not aware of any studies showing that one test or the other is better in a specific age group although very young children cannot perform the FVC manoeuvre. However, in patients who can perform the FVC manoeuvre, $\mathrm{FEV}_{1} \%$ predicted and the FEV $1 / \mathrm{FVC}$ ratio are reliable measurements of obstruction. According to the National Asthma Education and Prevention Program guidelines [8], the normal FEV1/FVC ratio is age-dependent: it should be $>80 \%$ for people aged $20-39$ years, $>75 \%$ for those aged $40-59$ years and $>70 \%$ for $60-80$-year-old patients.

$a$

9. Could you please provide some guidance on the use of hypertonic saline as a provocative test in patients with asthma but normal volume on spirometry and no significant postbronchodilator response?

M. Saba, Isfahan, Iran

A

9. In the European Respiratory Society Task Force report on indirect challenge [9], it is stated that bronchial responsiveness to hypertonic saline challenge correlates better with serum markers of inflammation than bronchial responsiveness to methacholine. A challenge with hypertonic saline is easy to perform and allows construction of a dose-response curve. The major 


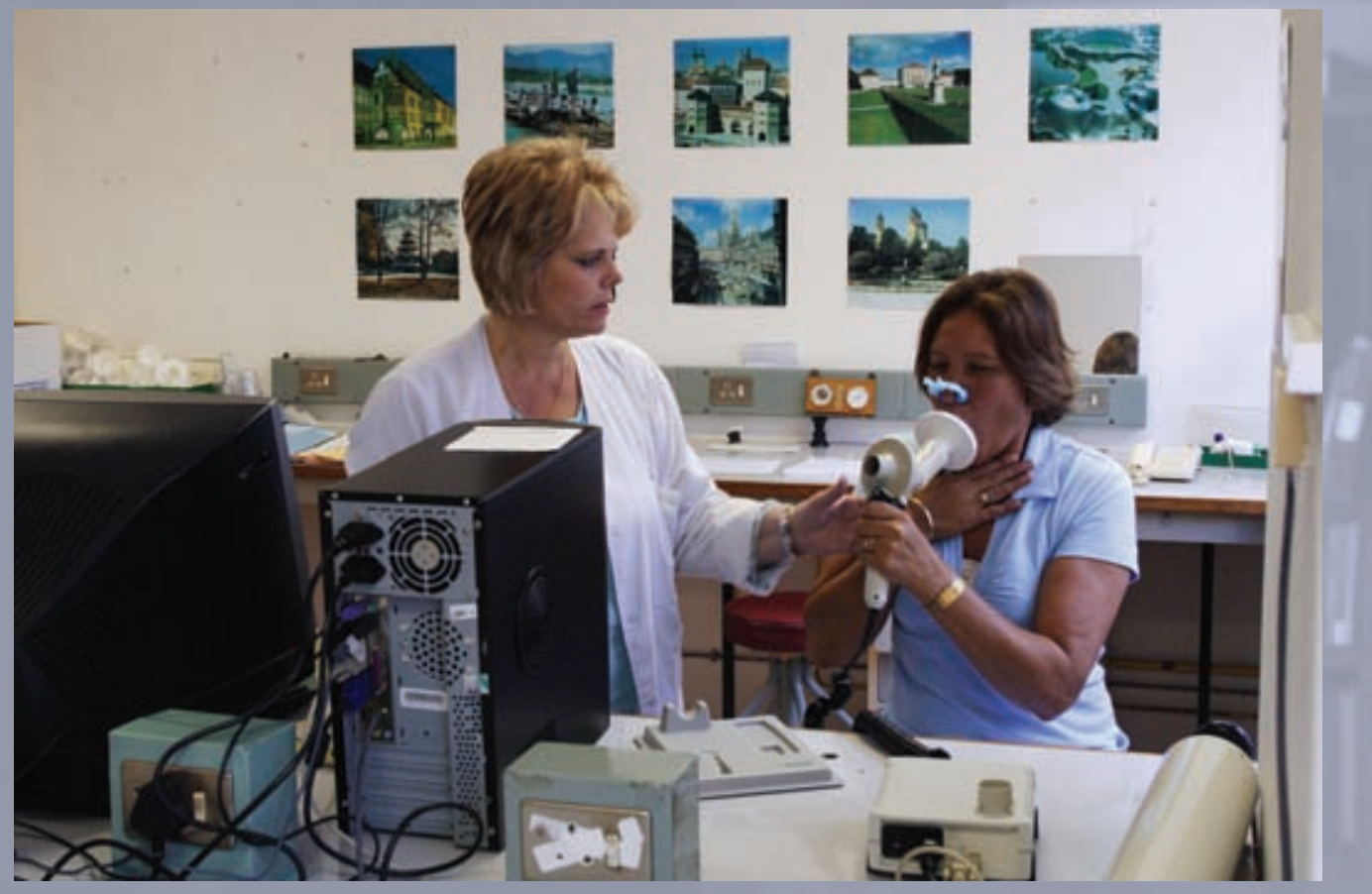

indications for using hypertonic aerosols or mannitol are to identify bronchial hyperresponsiveness consistent with active asthma or exercise-induced asthma and to evaluate bronchial responsiveness that will respond to treatment with anti-inflammatory drugs. A test using a hypertonic aerosol is an alternative to exercise or hyperventilation to identify patients with exercise-induced bronchospasm (EIB). Although some patients can have EIB and be negative to hypertonic saline or mannitol, this is unusual and has only been found in patients with very mild EIB. A challenge with a hypertonic aerosol may also be indicated in people with cough-variant asthma and may be indicated in pregnancy when a patient chooses not to be challenged with a pharmacological agent. Finally, bronchial hyperresponsiveness to hypertonic saline improves more than bronchial responsiveness to histamine after a course of inhaled corticosteroids. Hypertonic challenge may be used for assessing both acute and chronic treatment with corticosteroids.

From the literature, airway challenge with hypertonic saline (4.5\%) is performed according to the protocol approved by the International Study of Asthma and Allergies in Childhood steering committee [9]. The protocol comprises inhalation of $4.5 \%$ hypertonic saline aerosol generated by an ultrasonic nebuliser closed system for $0.5,1,2,4$ and 8 min (total $15.5 \mathrm{~min}$ ). After each dose step, two reproducible measurements of $F E V 1$ are assessed, of which the higher is selected. If FEV 1 falls 10-15\% compared with pre-challenge FEV 1 , the same dose step is repeated. The test stops after a total inhalation time of $15.5 \mathrm{~min}$, or if a drop in FEV 1 of $15 \%$ is reached. Salbuta$\mathrm{mol}$ is administered to relieve airflow limitation or symptoms when needed.

10. Is there any evidence base for the use of sublingual immunotherapy in the management of bronchial asthma?

P. Swamy, Jabalpur, India

$\triangle$

10. Immunotherapy in asthma can only be instituted in patients with mild asthma and sensitivity to one or, at the most, two aeroallergens and symptoms that correlate to this sensitivity [10]. In patients with moderate to severe disease and/or multiple sensitisation the risk is high and results are poor. So it is in a fact a selected group of people who may benefit and then the cost-benefit, possible risk and requirement for close follow-up should be examined. Sublingual immunotherapy seems to have similar results to classic subcutaneous specific immunotherapy but again the same limitations apply and sublingual specific immunotherapy requires equally close monitoring. 


\section{References}

1. Payne DN, Balfour-Lynn IM, Biggart EA, Bush A, Rosenthal M. Subcutaneous terbutaline in children with chronic severe asthma. Pediatr Pulmonol 2002; 33: 356-361.

2. Miller JD, Cox G, Vincic L, Lombard CM, Loomas BE, Danek CJ. A prospective feasibility study of bronchial thermoplasty in the human airway. Chest 2005; 127: 1999-2006.

3. Cox G, Miller JD, McWilliams A, Fitzgerald JM, Lam S. Bronchial thermoplasty for asthma. Am J Respir Crit Care Med 2006; 173: 965-969.

4. Bumbacea D, Campbell D, Nguyen L, et al. Parameters associated with persistent airflow obstruction in chronic severe asthma. Eur Respir J 2004; 24: 122-128.

5. Chanez P, Wenzel SE, Anderson GP, et al. Severe asthma in adults: what are the important questions? J Allergy Clin Immunol 2007; 119: 1337-1348.

6. ten Brinke A, Sterk PJ, Masclee AA, et al. Risk factors of frequent exacerbations in difficult-to-treat asthma. Eur Respir J 2005; 26: 812-818.

7. The ENFUMOSA Cross-Sectional European Multicentre Study of the Clinical Phenotype of Chronic Severe Asthma. European Network for Understanding Mechanisms of Severe Asthma. Eur Respir J 2003; 22: 470-477.

8. National Heart, Lung and Blood Institute, National Asthma Education and Prevention Program Expert Panel Report 3: Guidelines for the Diagnosis and Management of Asthma Full Report 2007. www.nhlbi.nih.gov/guidelines/asthma/ index.htm Date created: 2007.

9. Joos GF, 0'Connor B, Anderson SD, et al. Indirect airway challenges. Eur Respir J 2003; 21: 1050-1068.

10. Weiland SK, Björkstén B, Brunekreef B, Cookson WO, von Mutius E, Strachan DP. Phase II of the International Study of Asthma and Allergies in Childhood (ISAAC II): rationale and methods. Eur Respir J 2004; 24: 406-412.

\section{Useful resources}

The Asthma Control Test can be accessed at http:/ /www.qualitymetric.com/demos/TP_Launch.aspx?SID=52461

The Asthma Control Questionnaire is available from

http:/ / www.qoltech.co.uk/acq.html free of charge for non-commercial clinical practice or research

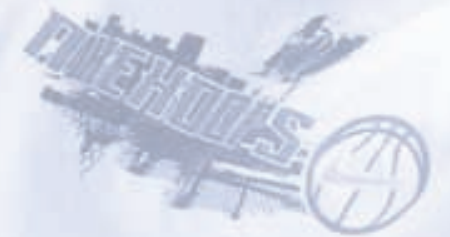

